\title{
Adaptive Mutation Rate for the Artificial Bee Colony Algorithm: A Case Study on Benchmark Continuous Optimization Problems
}

\author{
Syeda Shabnam Hasan \\ Department of Computer Science and Engineering \\ Ahsanullah University of Science and Technology \\ Dhaka-1208, Bangladesh
}

\begin{abstract}
A major problem with the Artificial Bee Colony (ABC) algorithm is its premature convergence to the locally optimal points of the search space, which often originates from the lack of explorative search capability of its mutation operator. This paper introduces $\mathrm{ABC}$ with Adaptive Mutation Rate (ABC-AMR), a novel algorithm that modifies the basic mutation operation of the original $\mathrm{ABC}$ algorithm in an explorative way. The novelty of the proposed algorithm lies in an adaptive mutation strategy that enables ABC-AMR to automatically adjust the mutation rate, separately for each candidate solution of the bee population, in order to customize the degree of explorations and exploitations around each candidate solution, while the original $\mathrm{ABC}$ algorithm employs a naïve fixed mutation rate. Besides, a few more explorative schemes and parameter values are employed by ABC-AMR to assist the adaptive mutation procedure. ABC-AMR is evaluated on several benchmark numerical optimization problems and results are compared with the basic $\mathrm{ABC}$ algorithm. Results show that ABC-AMR can perform better optimization than the original $\mathrm{ABC}$ algorithm on some of the benchmark problems.
\end{abstract}

\section{Keywords}

Artificial bee colony algorithm; Mutation; Exploration and exploitation; Continuous optimization.

\section{INTRODUCTION}

The Artificial Bee Colony (ABC) algorithm [1] is a recently introduced swarm intelligence based algorithm inspired by the intelligent food foraging behavior of the honey bees found in nature. Since its advent [2], ABC and its variants have often successfully employed to wide and diverse range of problems, such as numeric optimization [3], discrete optimization [4], multi-objective optimization [5], industrial process control [6], structural design [7], design of digital IIR filters [8], PID controller [9], machine learning [10] and so on [11].

In comparison to other greedy and local search based algorithms, ABC is more resilient against premature convergence and local optima, because the population of candidate solutions can maintain some amount of diversity that is necessary to continue search space explorations avoiding the locally optimal points. However, it is still possible (e.g., Refs. [12] - [14]) that the evolving population of candidate solutions loses its diversity and explorative search capability too soon. This leads the candidate solutions to prematurely get trapped around the local optima. The risk of premature convergence usually rises with reduced explorations and increased exploitations. But, increasing the explorations may lead to unacceptably slow convergence speed. So an adaptive and balanced mix of explorations and exploitations is often necessary for good results and sufficient convergence speed of the algorithm.

There exist a number of research works (e.g., Refs. [15] [34]) that attempt to alter the explorative and/or exploitative properties of the basic ABC algorithm. However, most of them focus on altering the selection operation only. In the literature, not much has been reported to improve the basic, non-adaptive and fixed mutation operator of $\mathrm{ABC}$. The proposed algorithm - $\mathrm{ABC}$ with Adaptive Mutation Rate (ABC-AMR) alters the mutation operation of $\mathrm{ABC}$, as well as incorporates few more basic schemes to increase the degree of explorations of the basic $\mathrm{ABC}$ algorithm. Unlike $\mathrm{ABC}$, the number of parameters that are mutated by $A B C-A M R$ is gradually self-adapted, cycle (i.e., generation) by cycle, separately for each candidate solution of the bee population. The objective is to customize the degree of explorations and exploitations at the individual candidate solution level, separately for each candidate solution during its mutation operation by adapting and adjusting its mutation rate separately.

The rest of this paper is organized as follows. Section 2 describes the $A B C$ algorithm. Section 3 presents a few improved $\mathrm{ABC}$-variants and explains how $\mathrm{ABC}-\mathrm{AMR}$ is significantly different from them. Section 4 describes ABC-AMR in details. Section 5 provides details of the benchmark problems and compares the results of different algorithms. Finally, section 6 leaves a few suggestions for further research with $\mathrm{ABC}-\mathrm{AMR}$

\section{THE ARTIFICIAL BEE COLONY (ABC) ALGORITHM}

Honey bees in a colony show remarkable self-organization and co-ordination skills in their food foraging behavior. Bees have to forage over a vast area in search of good sources of food. After an initial exploration stage, more bees are employed to collect honey from the more profitable food sources whereas fewer bees are assigned to the less worthy food sources. Some scout bees are also assigned for exploration to find newer food sources. If the quality of a food source declines after some exploitation, this information is also shared with other bees so that fewer bees are now attracted to this source. After the quality of a food source falls below some threshold, the bees assigned to it abandon it. The foraging process is initiated by scout bees that start searching for flower patches suitable as food sources. Quality is usually 
measured as a combination of some values, such as quantity and density of sugar, ease of access, distance from the colony etc. After they return to the hive, those scout bees that found a patch with quality above some threshold, deposit their nectar and then go to the 'dance floor' to perform a dance known as the 'waggle dance'. This dance plays the key role to communicate information among the bees about the food sources. The waggle dance contains three pieces of information: i) the quality of the flower patch of this dancing bee, ii) the distance of the flower patch from the hive, iii) the direction from the hive that you have to travel in order to reach the flower. The 'onlooker' bees, waiting around the dance floor, observe the waggle dances of these 'employed' bees that have found good food sources and pick any one of them to become its 'follower' and collect nectar from its flower patch. The better a flower patch as a food source, the bigger is the number of follower bees along with its employed bee. However, if the patch is no longer good enough, it will not be advertised in the next waggle dance and the bees recruited for it as employed or follower bees will choose either to follow some other employed bee or start working as a scout bee to randomly explore the search space for finding new food source.

The $\mathrm{ABC}$ algorithm mimics the food foraging behavior of the honey bees with these three groups of bees: employed bees, onlookers and scouts. A bee working to forage a food source (i.e. solution) previously visited by itself and searching only around its vicinity is called an employed bee. Employed bees perform waggle dance to propagate information of its food source to other bees. A bee waiting around the dance floor to choose any of the employed bees to follow is called an onlooker. A bee randomly searching a search space for finding a food source is called a scout. For every food source, there is only one employed bee and a number of follower bees. The scout bee, after finding a good food source also becomes an employed bee. In ABC algorithm implementation, half of the colony is employed bees and the other half is the onlookers. Number of food sources (i.e., solutions) is equal to the number of employed bees. An employed bee whose food source is exhausted (i.e. solution has not improved after several attempts) becomes a scout. The detailed pseudocode is given below.

Step 1) Generate an initial population of $N$ individuals. Each individual is a food source (i.e. solution) and has $D$ attributes, where $D$ is the dimensionality of the problem.

Step 2) Evaluate the fitness of each individual.

Step 3) Each employed bee searches in the neighborhood of its current position to find a better food source. For each employed bee, generate a new solution, $\boldsymbol{v}_{i}$ around its current position, $\boldsymbol{x}_{\boldsymbol{i}}$ using (1).

$$
v_{i j}=x_{i j}+\varphi_{i j}\left(x_{i j}-x_{k j}\right)
$$

Here, $k \in\left\{1,2, \ldots, N_{e m p}\right\}$ and $j \in\{1,2, \ldots, D\}$ are randomly chosen indices. $N_{e m p}$ is the number of employed bees. $\Phi_{i j}$ is a uniform random number generated from the range $[-1,1]$.

Step 4) Compute the fitness of both $\boldsymbol{x}_{\boldsymbol{i}}$ and $\boldsymbol{v}_{\boldsymbol{i}}$. Apply greedy selection scheme to choose the better one.

Step 5) Calculate the selection probability, $P_{i}$ for each solution, $\boldsymbol{x}_{\boldsymbol{i}}$ and normalize the probability value by (2).

$$
P_{i}=f i t_{i} / \sum_{k=1}^{N} f i t_{k}
$$

Step 6) Assign each onlooker bee to a solution, $x_{i}$ at random with probability proportional to $P_{\mathrm{i}}$

Step 7) Produce new food positions (i.e. solutions), $v_{i}$ for each onlooker bee using the corresponding employed bee $\boldsymbol{x}_{i}$ by using (1).

Step 8) Evaluate the fitness of each employed bee, $x_{i}$ and its produced onlooker bee, $\boldsymbol{v}_{\boldsymbol{i}}$. Apply greedy selection scheme to keep the one with better fitness and discard the other.

Step 9) If a particular solution has not been improved over a number (say, 30) of cycles, then select it for abandonment. Replace it by placing a scout bee at a food source placed uniformly at random over the entire search space using (3), i.e., for $j=1,2, \ldots, D$

$$
x_{i j}=\min _{j}+\operatorname{rand}(0,1) *\left(\max _{j}-\min _{j}\right)
$$

Step 10) Keep track of the best food source position (solution) found so far.

Step 11) Check for termination. If the best solution found is acceptable or maximum number of iterations has elapsed, stop and return the best solution found so far. Otherwise go back to step 2 and repeat.

\section{EXISTING VARIANTS OF THE ABC ALGORITHM}

There exist several recent works (e.g., Refs. [15] - [34]) that try to tweak the explorative and/or exploitative properties of the basic $\mathrm{ABC}$ algorithm. For example, the cooperative $\mathrm{ABC}$ (CABC) algorithm [16] decomposes the search space into a number of subspaces and enforces more explorations by employing different bee colonies to explore the different subspaces. Another explorative variant - $\mathrm{ABC}$ with diversity strategy (DABC) [17] tries to preserve sufficient amount of diversity among the candidate solutions by switching between two different mutation schemes. Chaotic ABC (CHABC) [18] is another explorative $\mathrm{ABC}$-variant that uses dynamic chaotic sequence generators, instead of random number generators, to improve the explorative characteristics of the basic $\mathrm{ABC}$ algorithm. The explorative search capacity of $\mathrm{ABC}$ may also be improved by intelligent organization of the locally optimal points [19] and using the information of the global best solution, as in the Gbest-guided ABC (GABC) [20] algorithm. The Hooke Jeeves ABC (HJABC) [21] is another improved $\mathrm{ABC}$-variant that hybridizes the Hooke Jeeves pattern search technique with the basic $\mathrm{ABC}$ algorithm. The elitist $\mathrm{ABC}$ (EABC) [22] is a hybrid $A B C$ variant which hybridizes $A B C$ with two different local search operators to intensify the exploitations around the best solutions. Quan and Shi [23] reported improvement of the convergence speed by introducing an exploitative search iteration operator based on the fixed point theorem of contractive mapping. Qingxian and Haijun [24] employed the Boltzmann selection scheme and introduced an improved initialization scheme to improve the convergence speed. The hybrid crossover based ABC (CbABC) [25] is an exploitative variant that strengthens the exploitation phase of $\mathrm{ABC}$ by using a crossover operation. Another new ABC-variant - NABC [26] alters the search pattern of both employed and onlooker bees by searching around neighborhood of the best solutions. The JA-ABC [27] tries to improve average fitness of bee population by replacing 
poor solutions with mutations of the fittest solution, which makes it exploitative. Some other recently introduced ABC variants can be found in the Refs. [28]- [34], but each of them comes with some limitations, such as inadequate degree of explorations [28]-[30], poor exploitations [31], slower rate of convergence of the algorithm [32] and increased computational complexity [33], [34].

A major weakness of most existing ABC-variants (e.g., Refs. [16]-[34]) is that they do not consider the individual explorative/exploitative needs of the candidate solutions; rather they treat all the candidate solutions equally, employing some population-wide uniform strategy, identically on all candidate solutions. Another limitation is that they try to improve either the explorative (e.g., Refs. [16] - [19], [31][34]) or the exploitative (e.g., Refs. [20] - [30]) properties of the basic $\mathrm{ABC}$ algorithm. The explorative enhancements are usually based on more explorative mutation, selection and/or initialization (e.g., Refs. [18], [19]) or employing some technique to maintain more population diversity (e.g., Refs. [16], [17]), while the exploitative developments are usually based on increasing the local search operations around the best candidate solutions (e.g., Refs. [21] - [22], [26] - [27]). However, only a few (i.e., Refs. [17], [20], [27]) of these algorithms make some efforts, more or less, to balance between the explorative and exploitative improvements. But they often use some fixed, rather than adaptive, strategy to balance the explorations with exploitations. For example, a fixed threshold value $d_{l o w}$ by DABC [17], fixed control parameter value $C$ by GABC [20] and fixed replacement rate of poor solutions by JA-ABC [27].

ABC-AMR differs from all these algorithms in a number of ways. First, ABC-AMR customizes explorations and exploitations separately for every candidate solution $\boldsymbol{x}_{\boldsymbol{i}}$ by introducing and separately maintaining a control parameter $\boldsymbol{r}\left[\boldsymbol{x}_{\boldsymbol{i}}\right]$ for each $\boldsymbol{x}_{\boldsymbol{i}}$. Secondly, ABC-AMR adopts a self-adaptive (rather than fixed, as in Refs. [16], [17], [20], [27], [30]) technique to adaptively control the mutation rate for each candidate solution. Finally, ABC-AMR also employs a few basic techniques to induce more explorations that assist the adaptive mutation rate strategy for better performance.

\section{ABC WITH ADAPTIVE MUTATION RATE (ABC-AMR)}

The proposed variant, ABC-AMR is different from the original $\mathrm{ABC}$ algorithm in four different aspects. First, the major difference between $\mathrm{ABC}$ and $\mathrm{ABC}-\mathrm{AMR}$ is that $\mathrm{ABC}-\mathrm{AMR}$ alters the mutation equation (1) which is used by steps 3 and 7 of the original $\mathrm{ABC}$ algorithm for producing new candidate solutions from the existing ones. ABC perturbs only a single parameter of an existing candidate solution $\boldsymbol{x}_{\boldsymbol{i}}$ by using (1), which means $\mathrm{ABC}$ has a fixed mutation rate of $1 / D$. In contrast, $\mathrm{ABC}-\mathrm{AMR}$ employs a self-adaptive scheme to automatically adapt the mutation rate at the individual solution level. The procedure is further explained in the paragraph that follows. Second, ABC-AMR employs a larger interval of $[-2,2]$ to randomly produce the $\varphi_{i j}$ values in (1) instead of the narrower $[-1,1]$ interval used by the original $\mathrm{ABC}$ algorithm. Third, to increase the degree of explorations, ABC-AMR employs three scout bees instead of only one scout used by the original $\mathrm{ABC}$ algorithm. Fourth, if a particular bee $\boldsymbol{x}_{\boldsymbol{i}}$ is not improved over the last 30 cycles, ABC-AMR first tries to improve it by applying crossover operation between $\boldsymbol{x}_{\boldsymbol{i}}$ and the best employed bee found so far, before abandoning it by scout bees. The second and third schemes improve the degree of explorations, while the fourth scheme increases degree of exploitations. All these four schemes work together to facilitate more effective mutations to produce better offspring solutions from the existing ones.

ABC-AMR includes a mutation probability $r\left[x_{i}\right]$ within each candidate solution $\boldsymbol{x}_{\boldsymbol{i}}$ which is gradually self-adapted, cycle by cycle, separately for each $\boldsymbol{x}_{\boldsymbol{i}}$. The original ABC algorithm perturbs only a single, random parameter of the existing candidate solutions using (1). This performs search along one dimension at a time, which may be suitable for separable problems, but inappropriate for complex non-separable problems. In contrast, ABC-AMR can perturb any number of parameters allowing search along any possible direction. To accomplish this, ABC-AMR maintains and automatically adapts a control parameter $r\left[x_{i}\right]$, separately for every candidate solution $\boldsymbol{x}_{\boldsymbol{i}}$. This parameter controls the mutation rate during producing trial solution $\boldsymbol{v}_{\boldsymbol{i}}$ from $\boldsymbol{x}_{\boldsymbol{i}}$. To perform self-adaptation of the value of $r\left[x_{i}\right]$, ABC-AMR does the following - before perturbing any parameter of $\boldsymbol{x}_{\boldsymbol{i}}$ during producing $\boldsymbol{v}_{i}$, the value of $r\left[x_{i}\right]$ is perturbed first, with probability $t$, using (4). This (possibly) perturbed value of $r$ is inherited by $\boldsymbol{v}_{\boldsymbol{i}}$, which is now referred as $r\left[\boldsymbol{v}_{\boldsymbol{i}}\right]$ and is used as the probability of perturbing the parameters of $\boldsymbol{x}_{\boldsymbol{i}}$ to produce $\boldsymbol{v}_{\boldsymbol{i}}$ from $\boldsymbol{x}_{\boldsymbol{i}}$. A more effective value of $r\left[\boldsymbol{v}_{\boldsymbol{i}}\right]$ is likely to produce fitter new solutions, which are likely to survive better than $\boldsymbol{x}_{i}$ and produce better, newer solutions that will propagate the better values of the mutation probability $r\left[\boldsymbol{v}_{\boldsymbol{i}}\right]$ across the population. Thus a gradual self-adaptation towards better, more effective mutation rates will take place across the population.

$$
\boldsymbol{r}\left[\boldsymbol{v}_{\boldsymbol{i}}\right]= \begin{cases}r_{\min }+\operatorname{rand}(0,1) *\left(r_{\max }-r_{\min }\right) ; & \text { if } \operatorname{rand}(0,1)<t \\ \boldsymbol{r}\left[\boldsymbol{x}_{i}\right] & \text { otherwise }\end{cases}
$$

Here, $t$ is the probability that $r\left[x_{i}\right]$ is perturbed first before perturbing any parameter of $\boldsymbol{x}_{i}$ during producing $\boldsymbol{v}_{i}$ from $\boldsymbol{x}_{\boldsymbol{i}}$. For all of our experiments, we have set $r_{\max }=1.0, r_{\min }=1 / D$ and $t=0.05$.

\section{EXPERIMENTAL STUDIES}

ABC-AMR is evaluated using a standard benchmark suite on numerical optimization problems consisting of 30 functions [1], [2], [18]. Table 1 presents a brief overview on each of the 30 standard benchmark functions. More details on each benchmark function can be found in [1]. The benchmark suite consists both unimodal $\left(f_{1}-f_{9}\right)$ and multimodal $\left(f_{10}-f_{30}\right)$, separable (e.g., $f_{1}, f_{3}, f_{15}, f_{16}$ ) and non-separable (e.g., $f_{2}, f_{4}, f_{14}$, $\left.f_{17}\right)$, high $\left(f_{1}-f_{18}\right)$ and low $\left(f_{19}-f_{30}\right)$ dimensional functions. To optimize a multimodal function, the search algorithm must possess both exploitative and explorative characteristics so that it can explore the locally optimal points without being trapped around any of them. Some of the multimodal functions can have hundreds of local minima, even when the dimensionality is just two or three. The number of local optima usually increases exponentially with the number of dimensions, which makes their optimization extremely difficult. For example, the Ackley function $f_{13}$ has one narrow global minimum basin, but with exponentially many minor local minima. The Griewank function $f_{14}$ has a component creating linkage among the variables, which complicates the search by perturbing any subset of the variables. The difficulty for the Schwefel function $f_{12}$ arises from its deep 
Table 1: Standard benchmark functions. $D$ : dimensionality, $S$ : $\operatorname{search}$ space, $f_{\text {min }}$ : function value at global minimum, $C$ : function characteristics - $U$ : Unimodal, $M$ : Multimodal, $S$ : Separable, $N$ : Non-separable.

\begin{tabular}{|c|c|c|c|c|c|}
\hline No & Function & $C$ & $D$ & $S$ & $f_{\min }$ \\
\hline$f_{1}$ & Sphere & $U S$ & 30 & {$[-100,100]^{D}$} & 0 \\
\hline$f_{2}$ & Schwefel 2.22 & $U N$ & 30 & {$[-10,10]^{D}$} & 0 \\
\hline$f_{3}$ & Schwefel 2.21 & $U S$ & 30 & {$[-10,10]^{D}$} & 0 \\
\hline$f_{4}$ & Schwefel 1.2 & $U N$ & 30 & {$[-100,100]^{D}$} & 0 \\
\hline$f_{5}$ & Powell & $U N$ & 24 & {$[-4,5]^{D}$} & 0 \\
\hline$f_{6}$ & Dixon-Price & $U N$ & 30 & {$[-10,10]^{D}$} & 0 \\
\hline$f_{7}$ & Rosenbrock & $U N$ & 30 & {$[-30,30]^{D}$} & 0 \\
\hline$f_{8}$ & Step & $U S$ & 30 & {$[-100,100]^{D}$} & 0 \\
\hline$f_{9}$ & Quartic & $U S$ & 30 & {$[-1.28,1.28]^{D}$} & 0 \\
\hline$f_{10}$ & Rastrigin & $M S$ & 30 & {$[-5.12,5.12]^{D}$} & 0 \\
\hline$f_{11}$ & $\begin{array}{l}\text { Non-continuous } \\
\text { Rastrigin }\end{array}$ & $M S$ & 30 & {$[-5.12,5.12]^{D}$} & 0 \\
\hline$f_{12}$ & Schwefel 2.26 & $M S$ & 30 & {$[-500,500]^{D}$} & -12569.5 \\
\hline$f_{13}$ & Ackley & $M N$ & 30 & {$[-32,32]^{D}$} & 0 \\
\hline$f_{14}$ & Griewank & $M N$ & 30 & {$[-600,600]^{D}$} & 0 \\
\hline$f_{15}$ & Alpine & $M S$ & 30 & {$[-10,10]^{D}$} & 0 \\
\hline$f_{16}$ & Weierstrass & $M S$ & 30 & {$[-0.5,0.5]^{D}$} & 0 \\
\hline$f_{17}$ & Penalized & $M N$ & 30 & {$[-50,50]^{D}$} & 0 \\
\hline$f_{18}$ & Penalized2 & $M N$ & 30 & {$[-50,50]^{D}$} & 0 \\
\hline$f_{19}$ & Foxholes & $M S$ & 2 & {$[-65.53,65.53]^{D}$} & 1 \\
\hline$f_{20}$ & Kowalik & $M N$ & 4 & {$[-5,5]^{D}$} & $3.07 \mathrm{e}-04$ \\
\hline$f_{21}$ & $\begin{array}{c}\text { Six Hump } \\
\text { Camel Back }\end{array}$ & $M N$ & 2 & {$[-5,5]^{D}$} & -1.0316 \\
\hline$f_{22}$ & Branin & $M S$ & 2 & {$[-5,10] \times[0,15]$} & 0.398 \\
\hline$f_{23}$ & Hartman3 & $M N$ & 3 & {$[0,1]^{D}$} & -3.86 \\
\hline$f_{24}$ & Hartman6 & $M N$ & 6 & {$[0,1]^{D}$} & -3.32 \\
\hline$f_{25}$ & Shekel5 & $M N$ & 4 & {$[0,10]^{D}$} & -10.15 \\
\hline$f_{26}$ & Shekel7 & $M N$ & 4 & {$[0,10]^{D}$} & -10.40 \\
\hline$f_{27}$ & Shekel10 & $M N$ & 4 & {$[0,10]^{D}$} & -10.54 \\
\hline$f_{28}$ & Fletcher-Powell & $M N$ & 10 & {$[-\pi, \pi]^{D}$} & 0 \\
\hline$f_{29}$ & Michalewicz & $M S$ & 10 & {$[0, \pi]^{D}$} & -9.66 \\
\hline$f_{30}$ & Langerman & $M N$ & 10 & {$[0,10]^{D}$} & -1.4 \\
\hline
\end{tabular}


Table 2: Performance of the proposed algorithm ABC-AMR, compared to the basic ABC algorithm on the benchmark functions. Results are averaged over 50 independent runs. Better performance by ABC-AMR is marked with boldface font. In case the performance difference is not significant by $t$-Test with at least $95 \%$ level of confidence (i.e., $\alpha=0.95$ ), it is marked as "Similar".

\begin{tabular}{|c|c|c|c|c|c|c|}
\hline \multirow{2}{*}{ No } & \multirow{2}{*}{$f_{\min }$} & \multicolumn{2}{|c|}{$\mathbf{A B C}$} & \multicolumn{2}{|c|}{ ABC-AMR } & \multirow{2}{*}{$\begin{array}{l}\text { Better Performance } \\
(t \text {-Test with } \alpha=0.95)\end{array}$} \\
\hline & & Mean Error & Std. Dev. & Mean Error & Std. Dev. & \\
\hline$f_{1}$ & 0 & $3.58 \mathrm{e}-11$ & $8.14 \mathrm{e}-12$ & $4.15 \mathrm{e}-11$ & $3.89 \mathrm{e}-12$ & Similar \\
\hline$f_{2}$ & 0 & $1.04 \mathrm{e}-14$ & $5.33 \mathrm{e}-14$ & $1.87 \mathrm{e}-14$ & $7.80 \mathrm{e}-15$ & Similar \\
\hline$f_{3}$ & 0 & $9.37 \mathrm{e}+00$ & $3.22 \mathrm{e}+00$ & $8.65 \mathrm{e}+00$ & $1.95 \mathrm{e}+00$ & Similar \\
\hline$f_{4}$ & 0 & $2.75 \mathrm{e}-10$ & $2.49 \mathrm{e}-10$ & $3.09 \mathrm{e}-10$ & $8.71 \mathrm{e}-11$ & Similar \\
\hline$f_{5}$ & 0 & $2.50 \mathrm{e}+00$ & $9.25 \mathrm{e}-01$ & $8.26 \mathrm{e}+00$ & $8.33 \mathrm{e}-01$ & $\mathrm{ABC}$ \\
\hline$f_{6}$ & 0 & $6.67 \mathrm{e}-01$ & $8.74 \mathrm{e}-02$ & $5.01 \mathrm{e}-03$ & $1.69 \mathrm{e}-03$ & ABC-AMR \\
\hline$f_{7}$ & 0 & $2.75 \mathrm{e}+00$ & $8.08 \mathrm{e}-01$ & $3.17 \mathrm{e}+00$ & $6.24 \mathrm{e}-01$ & Similar \\
\hline$f_{8}$ & 0 & 0 & 0 & 0 & 0 & Similar \\
\hline$f_{9}$ & 0 & $8.61 \mathrm{e}-13$ & $7.07 e-13$ & $7.23 \mathrm{e}-13$ & $2.02 \mathrm{e}-13$ & Similar \\
\hline$f_{10}$ & 0 & $5.79 \mathrm{e}-15$ & $2.48 \mathrm{e}-15$ & $6.58 \mathrm{e}-15$ & $2.90 \mathrm{e}-15$ & Similar \\
\hline$f_{11}$ & 0 & $8.82 \mathrm{e}-09$ & $2.33 \mathrm{e}-09$ & $7.23 \mathrm{e}-09$ & $2.51 \mathrm{e}-09$ & Similar \\
\hline$f_{12}$ & -12569.5 & $3.49 \mathrm{e}+02$ & $1.18 \mathrm{e}+02$ & $1.06 \mathrm{e}+02$ & $3.26 \mathrm{e}+01$ & ABC-AMR \\
\hline$f_{13}$ & 0 & $3.08 \mathrm{e}-06$ & $3.96 \mathrm{e}-07$ & $6.03 e-08$ & $9.58 \mathrm{e}-09$ & ABC-AMR \\
\hline$f_{14}$ & 0 & $4.35 \mathrm{e}-08$ & $8.47 \mathrm{e}-09$ & $3.99 \mathrm{e}-08$ & $1.78 \mathrm{e}-08$ & Similar \\
\hline$f_{15}$ & 0 & $6.90 \mathrm{e}-06$ & $2.15 \mathrm{e}-06$ & $6.82 \mathrm{e}-06$ & $1.93 \mathrm{e}-06$ & Similar \\
\hline$f_{16}$ & 0 & $3.03 \mathrm{e}-02$ & $8.69 \mathrm{e}-03$ & $7.36 \mathrm{e}-02$ & $8.59 \mathrm{e}-03$ & $\mathrm{ABC}$ \\
\hline$f_{17}$ & 0 & $5.82 \mathrm{e}-08$ & $9.42 \mathrm{e}-09$ & $7.11 \mathrm{e}-12$ & $2.43 \mathrm{e}-12$ & ABC-AMR \\
\hline$f_{18}$ & 0 & $2.64 \mathrm{e}-03$ & $8.53 \mathrm{e}-04$ & $2.49 \mathrm{e}-03$ & $7.25 \mathrm{e}-04$ & Similar \\
\hline$f_{19}$ & 1 & 0.03 & 0.013 & 0.02 & 0.045 & Similar \\
\hline$f_{20}$ & $3.07 \mathrm{e}-04$ & $7.60 \mathrm{e}-05$ & $6.69 \mathrm{e}-06$ & $6.56 \mathrm{e}-05$ & $7.80 \mathrm{e}-06$ & Similar \\
\hline$f_{21}$ & -1.0316 & 0.00 & 0.00 & 0.00 & 0.00 & Similar \\
\hline$f_{22}$ & 0.398 & 0.00 & 0.00 & 0.00 & 0.00 & Similar \\
\hline$f_{23}$ & -3.86 & 0.00 & 0.00 & 0.00 & 0.00 & Similar \\
\hline$f_{24}$ & -3.32 & 0.00 & 0.00 & 0.00 & 0.00 & Similar \\
\hline$f_{25}$ & -10.15 & 0.30 & 0.11 & 0.28 & 0.06 & Similar \\
\hline$f_{26}$ & -10.40 & 0.02 & 0.0025 & 0.03 & 0.021 & Similar \\
\hline$f_{27}$ & -10.54 & 0.12 & 0.045 & 0.04 & 0.0051 & ABC-AMR \\
\hline$f_{28}$ & 0 & 8.05 & 2.89 & 7.95 & 1.46 & Similar \\
\hline$f_{29}$ & -9.66 & 0.00 & 0.00 & 0.00 & 0.00 & Similar \\
\hline$f_{30}$ & -1.4 & 0.54 & 0.18 & 1.07 & 0.24 & $\mathrm{ABC}$ \\
\hline
\end{tabular}


local minima which are far from the single global minimum. The low dimensional functions $f_{19}-f_{30}$ have fewer local minima, but they are well separated and distant, making them difficult to be explored without being trapped around them.

Table 2 presents the results of ABC-AMR with the basic $\mathrm{ABC}$ [1] algorithm. For the high dimensional functions $f_{1}-f_{18}$, the common parameters are set as - population size $S N=50$, maximum number of function evaluations $F E=100000$ and limit $=100$. For low dimensional $f_{19}-f_{30}, S N=100, F E=10000$ and limit $=10 * D$. Each algorithm made 50 independent runs on each function. The mean and standard deviation of the best found solutions from different runs are reported in Table 2. Following points summarize our observations on the results.

- Out of the 30 functions $f_{1}-f_{30}$, ABC-AMR performs better than $\mathrm{ABC}$ on five functions, while $\mathrm{ABC}$ performs better only on three functions. On the remaining 22 functions, their results are similar (i.e., the performance difference is not statistically significant in $t$-tests with at least $95 \%$ degree of confidence). Thus the overall performance of $\mathrm{ABC}-\mathrm{AMR}$ is better than $\mathrm{ABC}$.

- The unimodal functions $f_{1}-f_{9}$ and low dimensional functions $f_{19}-f_{30}$ are relatively easier to optimize. On these functions, the performance of $\mathrm{ABC}$ and $\mathrm{ABC}-\mathrm{AMR}$ are mostly similar.

- On the high dimensional multimodal functions $f_{10}-f_{18}$, which are the most complex function family for any algorithm, the performance of ABC-AMR is much better than $\mathrm{ABC}$. On almost all these functions, $\mathrm{ABC}-\mathrm{AMR}$ performs either better or equally well to the basic $\mathrm{ABC}$ algorithm. This indicates that more explorations, as performed by ABC-AMR, are necessary for good performance on these multimodal functions with exponentially many locally optimal points.

- In summary, $\mathrm{ABC}-\mathrm{AMR}$ is better suited than the basic $\mathrm{ABC}$ algorithm on more complex multimodal and high dimensional functions.

\section{CONCLUSION}

This paper introduces $\mathrm{ABC}-\mathrm{AMR}$ - a novel variant of the basic $\mathrm{ABC}$ algorithm and evaluates its performance on several standard benchmark functions. Results indicate that $\mathrm{ABC}-\mathrm{AMR}$ can perform better than $\mathrm{ABC}$ on more complex functions which require more search space explorations. There might be several possible ways to further improve ABC-AMR. Firstly, ABC-AMR uses a simple strategy to control the mutation rate. Some more sophisticated scheme, possibly parameterized by the current maturity of the search process, may improve the algorithm further. Secondly, ABC-AMR tries more to improve the explorations only. Putting some emphasis on exploitations, especially around the best-so-far candidate solutions, may further improve the results. Thirdly, the quality of the final solution might be improved further by using an efficient local searcher after the execution of ABC-AMR is over. Finally, ABC-AMR has been applied only on the benchmark continuous optimization problems. It would be interesting to study how well ABC-AMR performs on many other existing problems, especially the discrete and real world ones. Some interesting examples of discrete and real world problems on which ABC-AMR could be employed are industrial process control [6], machine learning [10], bioinformatics [35], data mining [36], telecommunications [37], engineering analysis and design [38] and many others [11].

\section{REFERENCES}

[1] D. Karaboga and B. Basturk, On the performance of artificial bee colony (ABC) algorithm, Applied Soft Computing 8 (1) (2008) 687-697.

[2] D. Karaboga, An idea based on honey bee swarm for numerical optimization, Erciyes University, Kayseri, Turkey, Technical Report-TR06, 2005.

[3] D. Karaboga and B. Akay, A comparative study of artificial bee colony algorithm, Applied Mathematics and Computation 214 (1) (2009) 108-132.

[4] S. Sobti and P. Singla, Solving travelling salesman problem using bee colony based approach, International Journal of Engineering Research and Technology 2 (6) (2013) 186-189.

[5] K. Naidu, H. Mokhlis and A.H.A. Bakar, Multiobjective optimization using weighted sum Artificial Bee Colony algorithm for Load Frequency Control, International Journal of Electrical Power and Energy Systems 55 (2) (2014) 657-667.

[6] R. Mukherjee, D. Goswami and S. Chakraborty, Parametric optimization of Nd:YAG laser beam machining process using artificial bee colony algorithm, Journal of Industrial Engineering, vol. 2013, Article ID 570250, 15 pages, 2013. DOI: 10.1155/2013/570250.

[7] H. Garg, Solving structural engineering design optimization problems using an artificial bee colony algorithm, Journal of Industrial and Management Optimization, 10 (3) (2014) 777-794.

[8] Z. Zhao, D. Yin and Y. Jiang, Improved bee colony algorithm based on knowledge strategy for digital filter design, International Journal of Computer Applications, 47 (2) (2013) 241-248.

[9] A. Mishra, A. Khanna, N. Singh and V. Mishra, Speed control of DC motor using bee colony optimization, Universal Journal of Electrical and Electronic Engineering 1 (3) (2013) 68-75.

[10] A. Karegowda and M. Darshan, Optimizing feed forward neural network connection weights using artificial bee colony algorithm, International Journal of Advanced Research in Computer Science and Software Engineering 3 (7) (2013) 452-454.

[11] A. Bolaji, A. Khader, M. Betar and M. Awadallah, Bee colony algorithm, its variants and applications: A survey, Journal of Theoretical and Applied Technology 47 (2) (2013) 434-459.

[12] T. Park and K. R. Ryu, A Dual population genetic algorithm for adaptive diversity control, IEEE Trans. Evolutionary Computation 14 (6) (2010) 865-884.

[13] R. K. Ursem, Diversity guided evolutionary algorithms, in Proc. 7th Int. Conf. Parallel Problem Solving from Nature (PPSN), 2002, pp. 462-474.

[14] J. Lampinen and I. Zelinka, On stagnation of the differential evolution algorithm, in Proc. 6th Int. Mendel Conf. Soft Computing, Brno, Czech Republic, 2000, pp. 76-83. 
[15] V. Tereshko, A. Loengarov, "Collective DecisionMaking in Honey Bee Foraging Dynamics", Comput. Inf. Sys. J., vol. 9, no. 3, pp. 1-7, 2005.

[16] M. Abd, A cooperative approach to the artificial bee colony algorithm, in Proc. IEEE Congress on Evolutionary Computation (CEC), 2010, pp. 1-5.

[17] W. Lee and W. Cai, A novel artificial bee colony algorithm with diversity strategy, in Proc. 7th Int. Conf. Natural Computation, 2011, pp. 1441-1444.

[18] B. Wu and S. Fan, Improved artificial bee colony algorithm with chaos, in Computer Science for Environmental Engineering and Eco-Informatics, Part I, Communications in Computer and Information Science, eds. Y. Yu, Z. Yu and J. Zhao, vol. 158, 2011, pp. 51-56.

[19] L. Fenglei, D. Haijun and F. Xing, The parameter improvement of bee colony algorithm in TSP problem, Science Paper Online, Nov. 2007.

[20] G. Zhu and S. Kwong, Gbest-guided artificial bee colony algorithm for numerical function optimization, Applied Mathematics and Computation 217 (7) (2010) 31663173.

[21] F. Kang, J. Li, Z. Ma and H. Li, Artificial bee colony algorithm with local search for numerical optimization, Journal of Software 6 (3) (2011) 490-497.

[22] E. Montes and R. Koeppel, Elitist artificial bee colony for constrained real-parameter optimization, in Proc. IEEE Congress on Evolutionary Computation, 2010, pp. $1-8$.

[23] H. Quan and X. Shi, On the analysis of performance of the improved artificial bee colony algorithm, in Proc. 4th Int. Conf. Natural Computation (ICNC), 2008, 654-658.

[24] F. Qingxian and D. Haijun, Bee colony algorithm for the function optimization, Science Paper Online, Aug. 2008.

[25] S. Kumar, V. Sharma and R. Kumari, A novel crossover based artificial bee colony algorithm for optimization, International Journal of Computer Applications 82 (8) (2013) 18-25.

[26] Y. Xu, P. Fan and L. Yuan, A simple and efficient artificial bee colony algorithm, Mathematical Problems in Engineering, vol. 2013, Article ID 526315, 9 pages, 2013. DOI: $10.1155 / 2013 / 526315$.

[27] N. Sulaiman, J. Saleh and A. Abro, A modified artificial bee colony (JA-ABC) optimization algorithm, in Proc. International Conference on Applied Mathematics and
Computational Methods in Engineering (AMCME) 2013, pp. 74-79.

[28] A. Abro and J. Saleh, Enhanced global-best artificial bee colony optimization algorithm, in Proc. $6^{\text {th }}$ European Symposium on Computer Modeling and Simulation, 2012, pp. 95-100.

[29] W. Gao, S. Liu and L. Huang, A global best bee colony algorithm for global optimization, Journal of Computational and Applied Mathematics 236 (11) (2012) pp. 2741-2753.

[30] W. Gao and S. Liu, A modified artificial bee colony algorithm, Computers and Operations Research 39 (3) (2012) pp. 687-697.

[31] $20 \mathrm{~W}$. Gao and S. Liu, Improved artificial bee colony algorithm for global optimization, Information Processing Letters 111 (17) (2011) pp. 871-882.

[32] G. Zhu and S. Kwong, Gbest-guided artificial bee colony algorithm for numerical function optimization, Applied Mathematics and Computation 217 (7) (2010) pp. 31663173.

[33] A. Abro and J. Saleh, An enhanced artificial bee colony optimization algorithm, Recent Advances in Systems Science and Mathematical Modelling, ed. D.S. Nikos Mastorakis, Valeriu Prepelita, 2012: WSEAS Press.

[34] A. Banharnsakun, T. Achalakul and B. Sirinaovakul, The best-so-far selection in artificial bee colony algorithm, Applied Soft Computing 11 (2) (2011) pp. 2888-2901.

[35] C. Lin and S. Su, Using an efficient bee colony algorithm for protein structure prediction, Int. Journal of Innovative Computing, Information and Control 8 (3b) (2012) 2049-2064.

[36] M. Abdulsalam and A. Bakar, A cluster based deviation detection using the artificial bee colony (ABC) algorithm, International Journal of Soft Computing 7 (2) (2012) 71-78.

[37] A. Ozen and C. Ozturk. "A novel modulation recognition technique based on artificial bee colony algorithm in the presence of multipath fading channels, in Proc. IEEE $36^{\text {th }}$ International Conference on Telecommunications and Signal Processing (TSP), 2013, pp. 239-243.

[38] B. Akay and D. Karaboga, Artificial bee colony algorithm for large scale problems and design optimization, Journal of Intelligent Manufacturing 23 (4) (2012), 1001-1014. 